\title{
Radiation-induced crosslinking: II. Effect on the crystalline and amorphous densities of polyethylene )
}

\author{
C. J. Chen, D. C. Boose and G. S. Y. Yeh \\ Departments of Chemical Engineering and Materials Engineering, Macromolecular Research Center, University of Michigan, \\ Ann Arbor, Michigan, USA
}

\begin{abstract}
Small-angle x-ray scattering (SAXS) was used to determine the structural changes in polyethylene induced by radiation. The changes in densities of the crystalline and amorphous phases, $\rho_{c}$ and $\rho_{a}$, were calculated after direct determination of the mean square density fluctuation $\left\langle\eta^{2}\right\rangle$. $\rho_{a}$ increases with increasing radiation dose for both linear and branched polyethylene. This accounts for the serious discrepancy between crystallinities determined from wide-angle $x$-ray scattering and density measurements. This study confirms our previous proposal that crosslinks occur primarily in the noncrystalline phase, most likely at the defects in the lateral grain boundary regions.
\end{abstract}

Key words: Crosslinking; radiation; polyethylene; density; crystallinity; d-spacings; phase densities; crosslink locations and $\underline{\text { mechanisms; }}$ defects; lateral grain boundaries.

\section{Introduction}

A previous paper [1] reported the results of a study of changes in the crystalline and amorphous densities, $\rho_{c}$ and $\rho_{a}$, for a branched polyethylene, BPE (Alathon 10) as a function of radiation dose. The results showed that $\rho_{a}$ increased with increasing radiation dose, whereas $\rho_{c}$ remained unchanged. It was suggested that crosslinks take place primarily in the non-crystalline phase. In that study, the SAXS intensity was obtained from a microdensitometric tracing of a photograph taken with a pinhole camera. The integrated intensity was approximated as being proportional to the mean-square density fluctuation $\left\langle\eta^{2}\right\rangle$ and the volume crystallinity $v_{c}$ was approximated by the weight crystallinity $w_{c}$. This paper reports the direct determination of $\left\langle\eta^{2}\right\rangle$ from SAXS intensity obtained using slit collimation for samples of both linear and branched polyethylene, as well as the use of derived equations for $\rho_{a}$ and $\rho_{c}$ that are direct functions of $\rho, w_{c}$ and $\left\langle\eta^{2}\right\rangle$.

The use of SAXS to determine the densities of the crystalline and amorphous phases of polymers is well known [2-7]. It has now been demonstrated to be applicable to an irradiated bulk BPE. Among the structural techniques that were used in our earlier study, SAXS intensity measurements were found to be by far the most sensitive in the detection of radiation-induced crosslinking effects. That study was carried out on a branched PE known to consist of small crystallites with substantial lateral grain boundary areas. A question arose if the observed effects were also typical of linear polyethylene, LPE, consisting of larger crystallites and well developed lamellae. A LPE was chosen for the present study, together with another BPE; the same Alathon 10 used primarily for comparative purposes.

\section{Theoretical background}

According to Debye and Bueche $[8,9]$, and Porod [10], the invariant $Q$ is defined as

$$
Q=\int_{0}^{\infty} m^{2} I(m) d m,
$$

*) Dedicated to Prof. Dr. W. Pechhold on the occasion of his 60th birthday. 
where $I$ is the SAXS intensity obtained from pinhole collimation, $m=2 a \sin \theta$, and $a$ is the distance between the sample and the plane of registration. $Q$ is independent of the arrangement of scattering units and depends only on the mean-square fluctuation of electron density $<\eta^{2}>$ :

$$
Q=k P_{0} d a<\eta^{2}>
$$

where $k=\left(i_{e} / 2 \pi\right) \lambda^{3} N^{2}=0.834 \times 10^{-2} . i_{e}$ is Thompson's constant $\left(7.9 \times 10^{-26}\right), \lambda$ is the wavelength of the $C u K_{\alpha}$ peak, $N$ is Loschmidt's number, $P_{0}$ is the primary energy, and $d$ is the sample thickness.

For a two-phase structure consisting of crystalline and amorphous phases with densities $\rho_{c}$ and $\left.\rho_{a r}<\eta^{2}\right\rangle$ is given by [10]

$$
<\eta^{2}>=v_{c} v_{a}\left(\rho_{c}-\rho_{a}\right)^{2},
$$

where $v_{c}$ and $v_{a}$ are the respective volume fractions. The density difference $\left(\rho_{c}-\rho_{a}\right)$ or $\Delta \rho$ can be obtained from $\left\langle\eta^{2}\right\rangle$ if the volume fractions are known.

In order to determine the crystalline and amorphous densities, $\rho_{c}$ and $\rho_{a}$, another relation is required:

$$
v_{c}=\left(\rho-\rho_{a}\right) /\left(\rho_{c}-\rho_{a}\right)=\left(\rho / \rho_{c}\right) w_{c} .
$$

Using weight crystallinity $w_{c}$ from WAXS,$<\eta^{2}>$ from SAXS, and the experimentally measured bulk density $\rho$, the values of $\rho_{c}$ and $\rho_{a}$ can be obtained according to the following derived equations from Eqs. (3) and (4):

$$
\begin{aligned}
& \rho_{c}=\rho+\frac{\left\langle\eta^{2}\right\rangle}{2 \rho w_{c}}+\left\{\frac{\left\langle\eta^{2}\right\rangle}{w_{c}}\left[\left(l-w_{c}\right)+\frac{\left\langle\eta^{2}\right\rangle}{4 \rho^{2} w_{c}}\right]\right\}^{1 / 2} \\
& \rho_{a}=\rho+\frac{\left\langle\eta^{2}\right\rangle}{2 \rho\left(l-w_{c}\right)}-\left\{\frac{\left\langle\eta^{2}\right\rangle}{\left(l-w_{c}\right)}\left[w_{c}+\frac{\left.<\eta^{2}\right\rangle}{4 \rho^{2}\left(l-w_{c}\right)}\right]\right\}^{1 / 2} .
\end{aligned}
$$

These equations are different from those used by others $[4,7]$, where they expressed $\rho_{c}$ and $\rho_{a}$ in terms of $v_{c}$ (then approximated by $w_{c}$ ), rather than $w_{c}$; their equations are relatively straight forward to derive.

\section{Experimental}

Two kinds of polyethylene, a BPE Alathon 10 and a LPE Alathon 7040 , were used in our experiments. The Alathon 10 samples were the same ones used in a previous study [1]. The BPE has a density of $0.92 \mathrm{~g} / \mathrm{cm}^{3}$ and a viscosity average molecular weight $M_{v}$ of 52 200. Alathon 7040 has a density of $0.95 \mathrm{~g} / \mathrm{cm}^{3}$ and a $M_{v}$ of 41500 . Sample preparations and irradiation methods have been described in the previous paper [1], along with procedures for gelation, density and WAXS measurements. Radiation doses range from 10 to 400 Mrad.

SAXS was conducted on a Rikagu-Denki camera with Ni-filtered $\mathrm{Cu} \mathrm{K}_{\alpha}$ radiation and slit collimation. Intensities were recorded with a proportional counter in connection with a pulse height discriminator. Step scans from 0.1 to 3.0 degrees $(2 \Theta)$ in increments of 0.05 degrees were made with intensity recorded by measuring the time per 5000 counts. This results in a probable counting error of less than $1 \%$ for each measurement [11]. The smeared SAXS intensity was corrected for slit length using Schmidt's program [12].

\section{Results}

Gel content increases with increasing radiation dose, shown in Fig. 1, for both Alathon 10 and Alathon 7040, indicating the formation of a network structure in the irradiated samples. Also shown in Figure 1 are plots of $1 / M_{c}$ against radiation dose. $M_{c}$ is the average molecular weight between crosslinks calculated from Flory-Huggins' equation [13]. Straight lines are obtained for both materials, indicating that the crosslink density is linearly proportional to the radiation dose in the range studied.

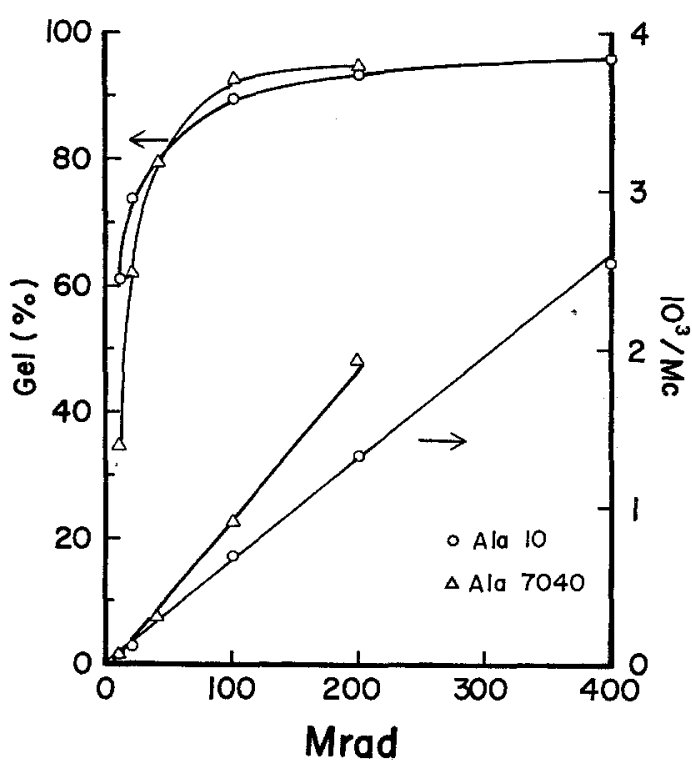

Fig. 1. Changes in gel content and $1 / M_{c}$ as a function of radiation dose for BPE Alathon 10 and LPE Alathon 7040 
Figure 2 shows changes in the bulk density with increasing radiation doses. Density increases slightly with increasing dose for both polyethylenes. Values of volume crystallinity $v_{c}$ calculated from these density data, using the conventional constant values of $\rho_{c}$ and $\rho_{a}$, are shown by dotted lines in Fig. 3 .

Using these constant values, crystallinity appears to increase slightly with radiation. In contrast, $w_{c}$ determined from WAXS, plotted in solid lines in Fig. 3 , shows a decrease, as generally expected for crosslinked polyethylene with increasing radiation. A similar crystallinity decrease with increasing radiation dose has also been observed from heat of fusion measurements [14]. These noticeable discrepancies can be resolved as we had done previously [1] by using crystalline $\rho_{c}$ and amorphous $\rho_{a}$ density values that have been corrected for the effects of radiation.

A typical slit-smeared SAXS curve is shown in Fig. 4 with error bars included. Collimation corrections for finite slit length were made using Schmidt's program [12]. Figure 5 shows both the slit-smeared and the corrected SAXS curves for unirradiated Alathon 10 . The correction not only sharpens the peak, but shifts its position toward a higher angle. The long periods calculated from peak positions are $220 \AA$ and

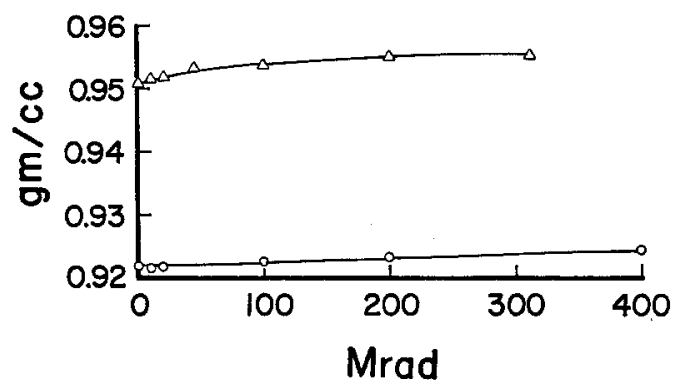

Fig. 2. Changes in density as a function of radiation dose for Alathon $10(\bigcirc)$ and $7040(\triangle)$

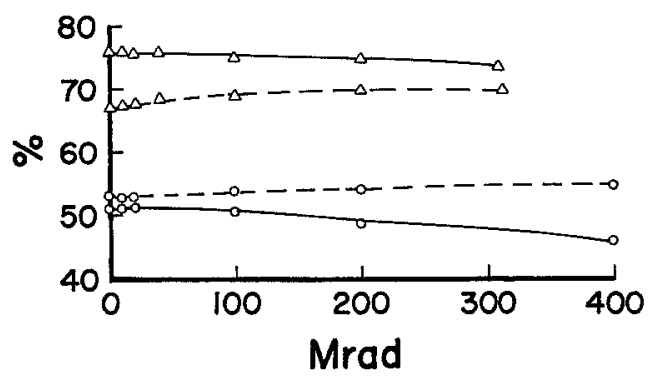

Fig. 3. Changes in crystallinities as a function of radiation dose for Alathon $10(O)$ and $7040(\triangle)$. Solid lines represent $w_{c}$ (from WAXS) and dotted lines represent $v_{c}$ (from density).

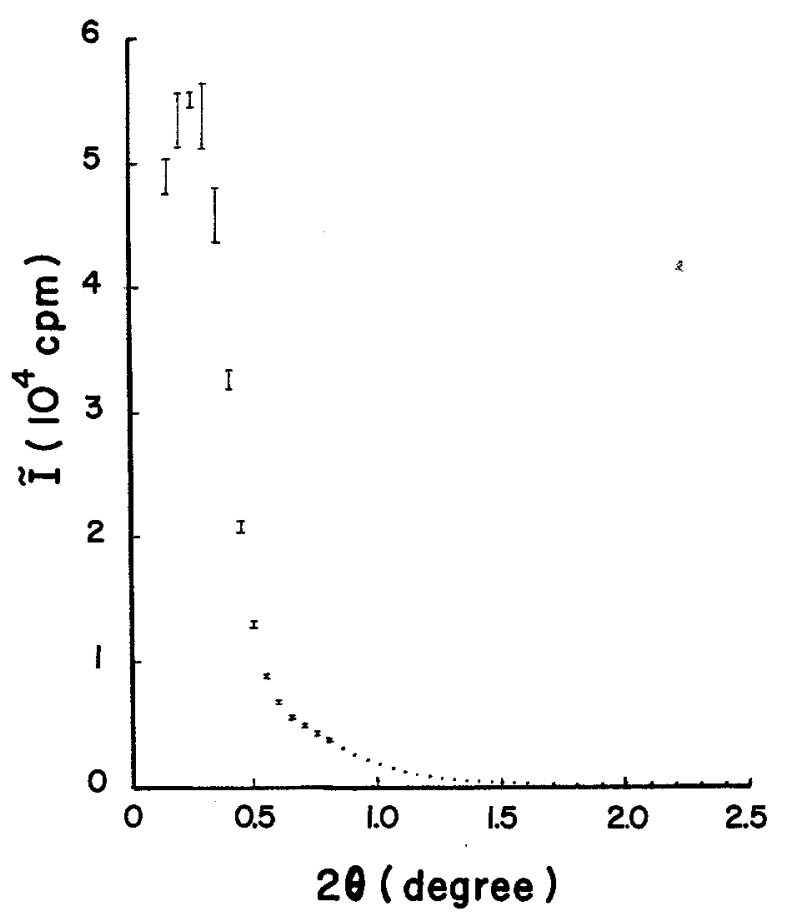

Fig. 4. Typical smeared SAXS curve for PE. Error bars are included.

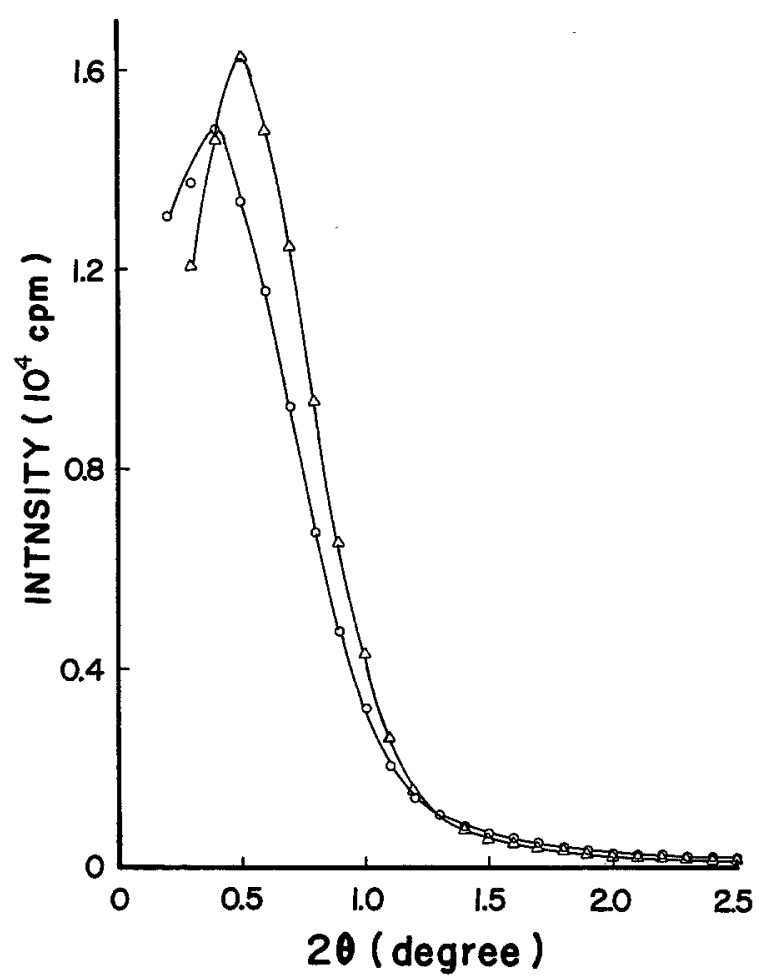

Fig. 5. SAXS curves of an unirradiated Alathon 10. Slit-smeared $(O)$ and desmeared $(\triangle)$ 
$170 \AA$ for smeared and corrected Alathon 10 , respectively. For Alathon 7040 the corresponding values are $325 \AA$ and $275 \AA$.

The slit-smeared SAXS curves of Alathon 10 and Alathon 7040 for various radiation doses are shown in Figs. 6 and 7. The peak intensity decreases with increasing radiation while the peak position remains unchanged, the latter indicating that the long period remains essentially constant with increasing degrees of crosslinking.

The SAXS intensity, corrected for finite slit length, was used to determine the invariant $Q$ according to Eq. (1). By partial integration we have

$$
\begin{aligned}
Q=\int_{0}^{\infty} m^{2} I(m) d m & =\int_{0}^{m *} m^{2} I(m) d m+ \\
& +\int_{m *}^{\infty} m^{2} I(m) d m
\end{aligned}
$$

The first part of the integral measures the area under the curve $m^{2} I(m)$ vs $m$ from 0 to $m^{*}\left(m^{*}\right.$ was $1.248 \mathrm{~cm}$ for this study). This area contributes $85-90 \%$ of the total $Q$ value for all samples. (The contribution of the unobserved part of the scattering curve at the smallest angles was estimated by extrapolating the $m^{2} I(m)$ curve down to $m=0$.) Using the relation $I(m) \alpha m^{4}$ for the tail of the scattering curve (derived

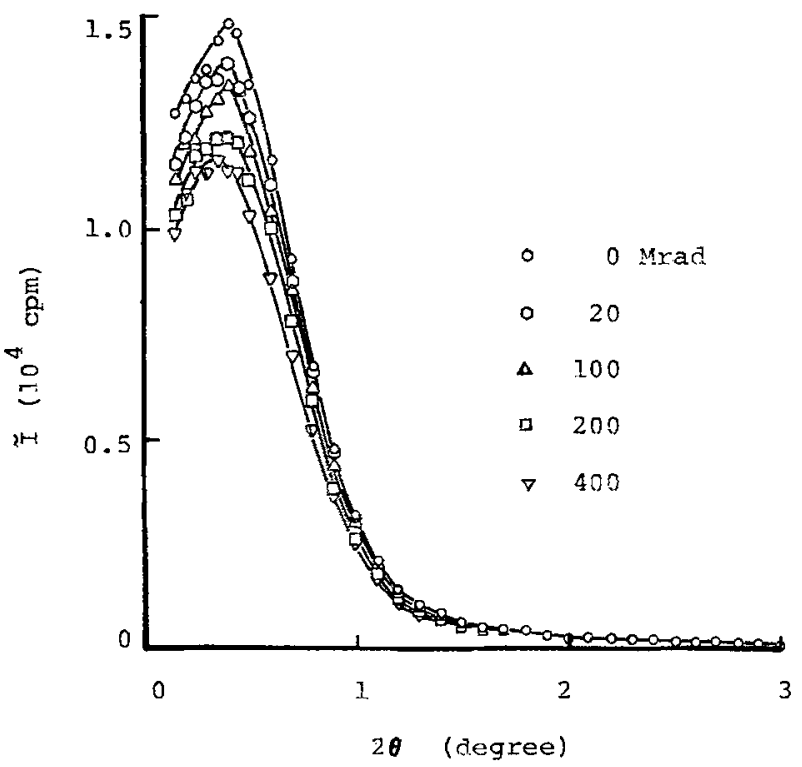

Fig. 6. SAXS curves of Alathon 10 with various radiation doses

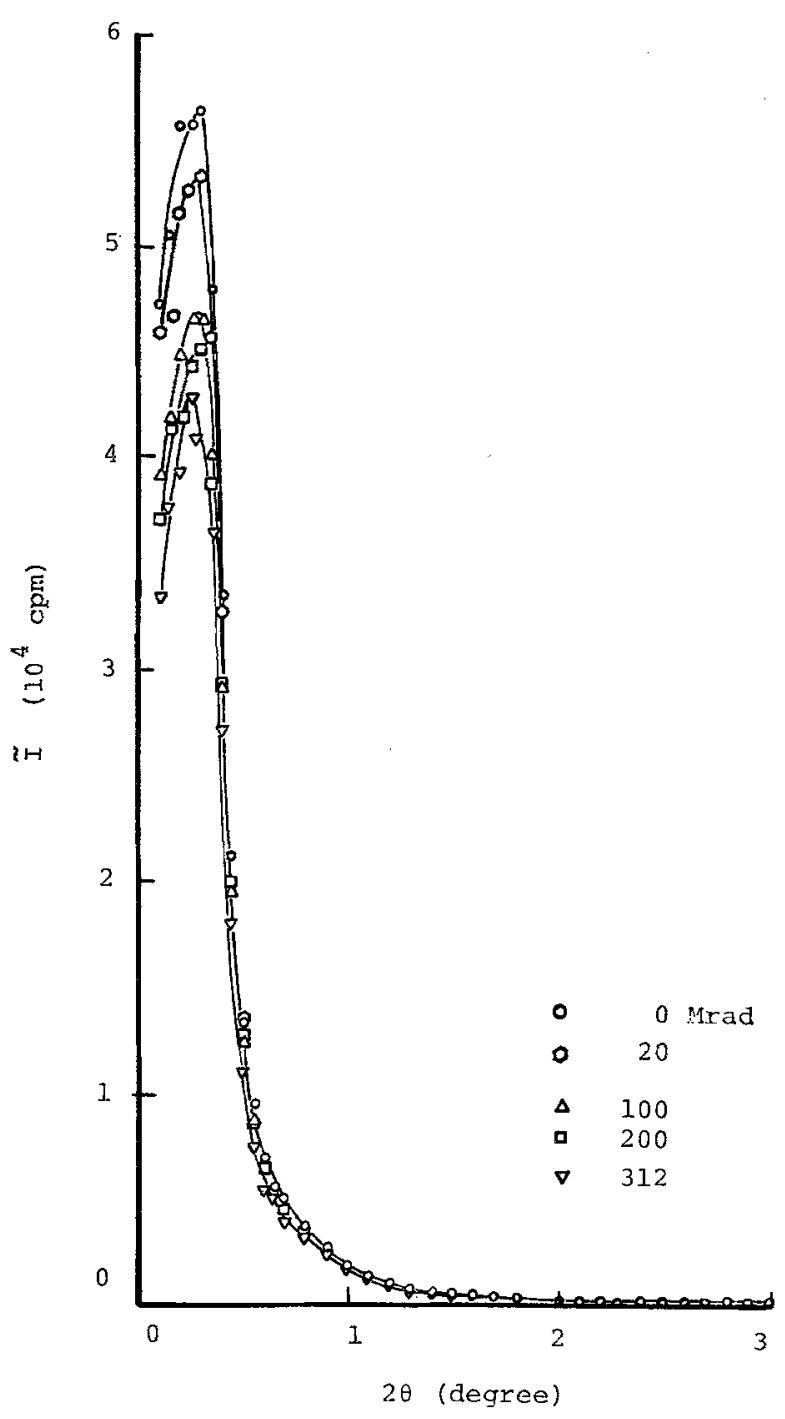

Fig. 7. SAXS curves of Alathon 7040 with various radiation doses

from Porod's theory [10]) the second integral becomes

$$
\int_{m^{*}}^{\infty} m^{2} I(m) d m=\int_{m^{*}}^{\infty} m^{2}(c / m)^{4} d m=c / m^{*},
$$

where $c$ is the intercept of a $m^{4} I(m)$ versus $m^{4}$ plot. The errors introduced by these simplifications are believed to be less than $10 \%$ [4]. Figure 8 shows plots of $m^{2} I(m)$ vs $m$ for BPE Alathon 10 samples.

Table 1 lists the $Q$ values of both polymers for various radiation doses. $Q$ is observed to decrease with increasing radiation, indicating a decrease in mean-square density fluctuation $\left\langle\eta^{2}\right\rangle$ with dose 
Table 1.

\begin{tabular}{|c|c|c|c|c|c|c|c|c|c|}
\hline Sample & Mrad & 0 & 10 & 20 & 40 & 100 & 200 & 312 & 400 \\
\hline \multirow[t]{2}{*}{ Alathon 10} & $\mathrm{Q}\left(\mathrm{cpm}-\mathrm{cm}^{3}\right)<\eta^{2}>\times 10^{3}$ & 1013.69 & 1011.43 & 920.75 & - & 893.20 & 783.19 & - & 718.06 \\
\hline & $\left(\text { mole-elec } / \mathrm{cm}^{3}\right)^{2}$ & 1.260 & 1.257 & 1.145 & - & 1.110 & 0.974 & - & 0.893 \\
\hline \multirow[t]{2}{*}{ Alathon 7040} & $Q\left(\mathrm{cpm}-\mathrm{cm}^{3}\right)<\eta^{2}>\times 10^{3}$ & 1220.64 & 1176.90 & 1157.35 & 1087.19 & 1061.87 & 1000.91 & 914.00 & - \\
\hline & $\left(\text { mole-elec } / \mathrm{cm}^{3}\right)^{2}$ & 1.517 & 1.463 & 1.439 & 1.351 & 1.320 & 1.244 & 1.136 & - \\
\hline
\end{tabular}

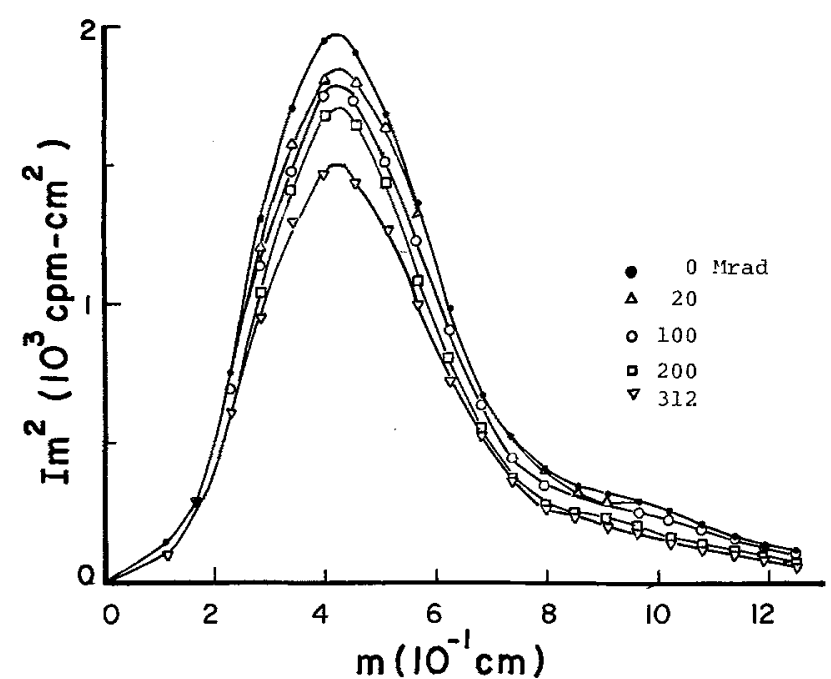

Fig. $8 . m^{2} I /(m)$ vs $m$ plots for Alathon 10 of various doses.

since $Q$ depends only on $\left\langle\eta^{2}\right\rangle$ (see Eq. (2)). The decrease in $\left\langle\eta^{2}\right\rangle$ amounts to $25-30 \%$ of the initial values at $300-400 \mathrm{Mrad}$, as shown in Fig. 9 and Table 1. $\left\langle\eta^{2}\right\rangle$ was obtained in absolute units (mole-electrons $\left./ \mathrm{cm}^{3}\right)^{2}$ using a calibrated Kratky sample. The $\left\langle\eta^{2}\right\rangle$ values are $1.260 \times 10^{-3}$ and $1.517 \times 10^{-3}$ for unirradiated branched and linear PE, respectively. These values are in excellent agreement with reported values $[4,15]$, being between $1.0 \times 10^{-3}$ and $1.88 \times 10^{-3}$.

The densities of the crystalline and amorphous phases, $\rho_{c}$ and $\rho_{a}$ (Fig. 10) were calculated from Eqs. (5) and (6) using $w_{c}$ from WAXS, $<\eta^{2}>$ from SAXS and $\rho$ from density gradient column measurements. Values of $\rho_{c}$ and $\rho_{a}$ are also listed with density, crystallinity, and $\left\langle\eta^{2}>\right.$ values in Tables 2 and 3 for Alathon 10 and Alathon 7040, respectively.

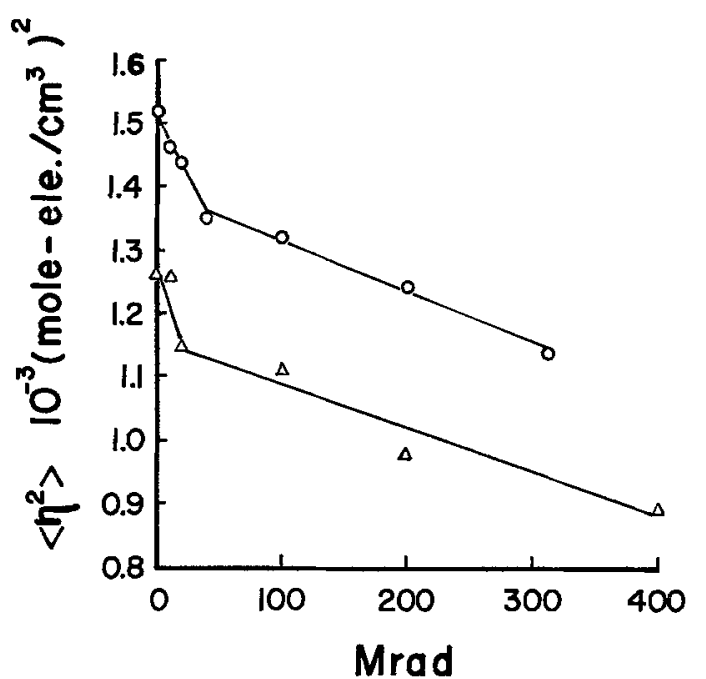

Fig. 9. Changes in mean-square density fluctuation as a function of radiation dose for Alathon $10(\triangle$ and $7040(O)$.

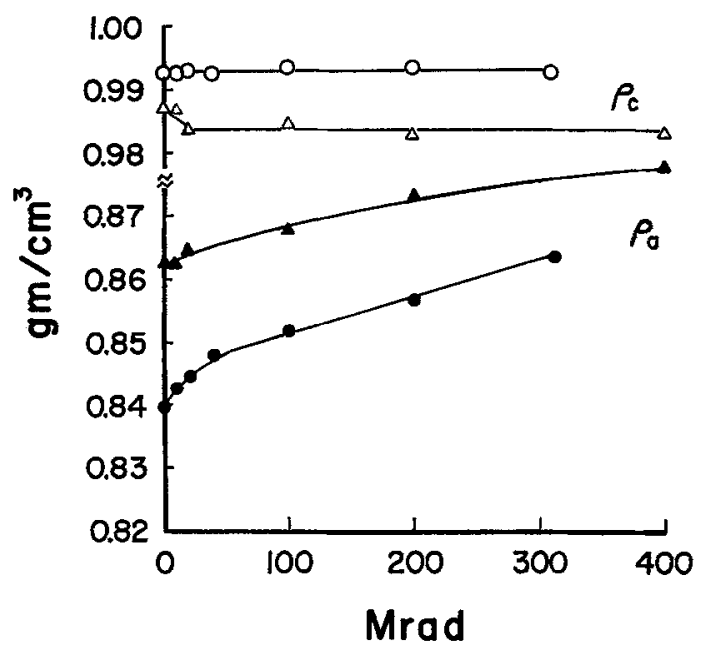

Fig. 10. Changes in crystalline and amorphous densities as a function of radiation dose for Alathon $10(\triangle$ and $(\Delta)$ and $70401 \bigcirc$ and ). 
Table 2.

\begin{tabular}{llllll}
\hline Mrad $\rho\left(\mathrm{g} / \mathrm{cm}^{3}\right)$ & $w_{c}(\%)$ & $\begin{array}{l}<\eta^{2}>\times 10^{3} \\
(\text { mole- } \\
\left.\text { elec } / \mathrm{cm}^{3}\right)^{2}\end{array}$ & $\rho_{c}\left(\mathrm{~g} / \mathrm{cm}^{3}\right)$ & $\rho_{a}\left(\mathrm{~g} / \mathrm{cm}^{3}\right)$ \\
\hline 0 & 0.9218 & 50.94 & 1.260 & 0.987 & 0.863 \\
10 & 0.9216 & 50.89 & 1.257 & 0.987 & 0.863 \\
20 & 0.9215 & 51.17 & 1.145 & 0.983 & 0.865 \\
100 & 0.9226 & 50.30 & 1.110 & 0.984 & 0.868 \\
200 & 0.9230 & 48.52 & 0.974 & 0.983 & 0.873 \\
400 & 0.9241 & 46.96 & 0.893 & 0.983 & 0.878 \\
\hline
\end{tabular}

Table 3.

\begin{tabular}{llllll}
\hline Mrad $\rho\left(\mathrm{g} / \mathrm{cm}^{3}\right)$ & $w_{c}(\%)$ & $\begin{array}{l}<\eta^{2}>\times 10^{3} \\
\text { (mole- } \\
\left.\text { elec } / \mathrm{cm}^{3}\right)^{2}\end{array}$ & $\rho_{c}\left(\mathrm{~g} / \mathrm{cm}^{3}\right)$ & $\rho_{a}\left(\mathrm{~g} / \mathrm{cm}^{3}\right)$ \\
\hline 0 & 0.9508 & 75.94 & 1.517 & 0.993 & 0.840 \\
10 & 0.9514 & 75.74 & 1.463 & 0.993 & 0.843 \\
20 & 0.9517 & 75.41 & 1.439 & 0.993 & 0.845 \\
40 & 0.9530 & 75.77 & 1.351 & 0.992 & 0.848 \\
100 & 0.9536 & 74.94 & 1.320 & 0.993 & 0.852 \\
200 & 0.9548 & 74.68 & 1.244 & 0.994 & 0.856 \\
312 & 0.9549 & 73.55 & 1.136 & 0.993 & 0.863 \\
\hline
\end{tabular}

$\rho_{c}$ remains essentially constant for both polymers, i.e., $0.983 \mathrm{~g} / \mathrm{cm}^{3}$ for Alathon 10 and $0.993 \mathrm{~g} / \mathrm{cm}^{3}$ for Alathon 7040. There appears to be a small decrease in $\rho_{c}$ at small doses for the BPE, though the magnitude of decrease is within the experimental error. In contrast, the amorphous density $\rho_{a}$ increases continuously with increasing radiation dose, from 0.863 $\mathrm{g} / \mathrm{cm}^{3}$ to $0.878 \mathrm{~g} / \mathrm{cm}^{3}$ for branched PE and from 0.840 to $0.863 \mathrm{~g} / \mathrm{cm}^{3}$ for linear PE. These results are in agreement with previous results on Alathon $10 \mathrm{ob}-$ tained by photographic methods [1].

\section{Discussion}

Again, one significant result from this study is the demonstration of SAXS for determining the structural changes induced by irradiation of PE. Interestingly, the changes can be seen in both LPE and BPE.

The mean-square density fluctuation $\left\langle\eta^{2}\right\rangle$ decreases by $25-30 \%$ of its original value with $300-$ $400 \mathrm{Mrad}$. This reduction is much greater than the estimated maximum experimental error of $10 \%$. Even for a dose of 100 Mrad there is a $12 \%$ decrease in $\left\langle\eta^{2}\right\rangle$. In comparison, WAXS, the most commonly used technique for structural determinations, cannot detect a significant change in that dose range. For example, crystallinity determined from WAXS remains almost constant up to $100 \mathrm{Mrad}$. We now consider in greater detail the various origins for the observed decrease in $\left\langle\eta^{2}\right\rangle$.

The lamellar structure of polymers is usually described as having a simple two-phase morphology, with crystalline and amorphous densities $\rho_{c}$ and $\rho_{a}$ considered constant, and having a sharply defined boundary between the phases. This simple model has been shown to adequately describe PE both crystallized from solution $[2,15]$ and from the melt $[4$, 15], but has been shown to be invalid for drawn PE [5] and for PET [7] (both drawn and undrawn). The validity of such a two-phase model with respect to irradiated PE may be checked by comparing the experimentally determined values of the mean-square density fluctuation $\left\langle\eta^{2}\right\rangle_{\text {exp }}$, taken from $Q$ values (by Eq. 2), with values calculated assuming a two-phase model with constant $\rho_{c}$ and $\rho_{a}$ densities:

$$
<\eta^{2}>_{c a l}=v_{c} v_{a}\left(\rho_{c}-\rho_{a}\right)^{2}
$$

The ratio of these two values, $\left\langle\eta^{2}\right\rangle_{\exp } /\left\langle\eta^{2}\right\rangle_{\text {cal }}$, should be approximately equal to 1 if the model is valid. A plot of these ratios as a function of radiation dose, shown in Fig. 11, reveals that the simple two-phase

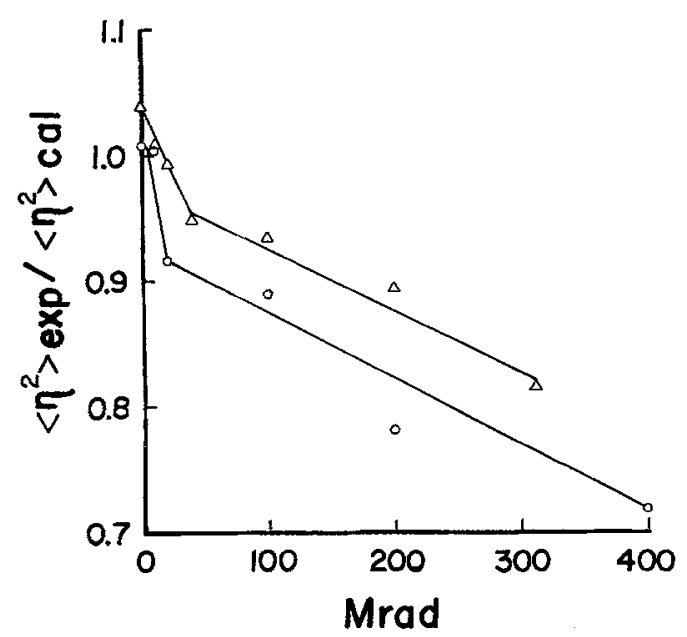

Fig. 11. Changes in the ratio of experimental to calculated mean square density fluctuations as a function of radiation dose for Alathon $10(\bigcirc$ and $7040(\triangle)$. 


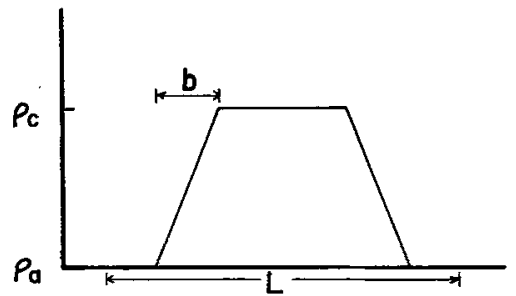

Fig. 12. Schematic representation of electron density distribution with a finite length of transition zone.

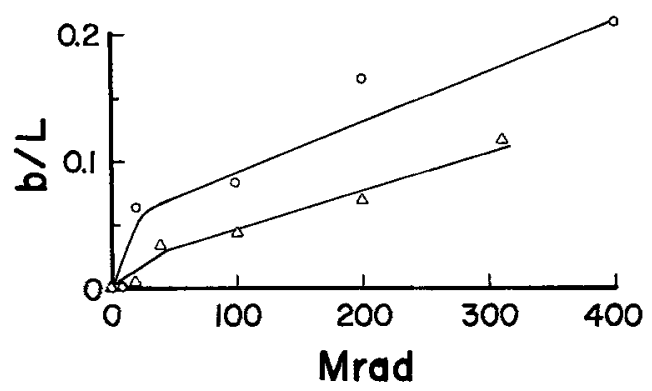

Fig. 13. Dependence of the ratio $b / L$ on radiation dose for Alathon $10(\bigcirc$ and $7040(\triangle)$.

model is a fair approximation for unirradiated PE, but the model is increasingly in error as the radiation. dose increases. Thus, the structure of irradiated PE cannot be described by the ideal two-phase model, as had already been implied in our previous study [1], as well as by the demonstration [1] that corrected $\rho_{c}$ and $\rho_{a}$ values must be used in order to obtain correct values of $v_{c}$.

The inadequacy of the simple two-phase model for irradiated PE may also imply that a transition region of finite width exists between the crystalline and amorphous phases.

For a system having a transition zone with a linear density gradient, as shown schematically in Fig. 12, Eq. (3) can be modified as follows $[16,17]$ :

$$
<\eta^{2}>=\left(\rho_{c}-\rho_{a}\right)^{2}\left[v_{a} v_{c}-b /(3 L)\right],
$$

where $b$ is the width of the transition zone and $L$ is the long period. The ratio $b / L$ can be calculated from the experimental value of $\left\langle\eta^{2}\right\rangle$ and volume fraction $v_{c}$, using the conventional values of $\rho_{c}$ and $\rho_{a}$. The ratio $b / L$, plotted in Fig. 13, shows an increase with increasing radiation for both polymers, from about 0 (at $0 \mathrm{Mrad}$ ) to 0.21 at $400 \mathrm{Mrad}$ for Alathon 10 and to 0.12 at $312 \mathrm{Mrad}$ for Alathon 7040 . These values correspond to a transition zone with a width of $36 \AA$ for Alathon $10(L=170 \AA)$ and $32 \AA$ for Alathon 7040 $(L=275 \AA)$.

This increase in the width of the transition zone with radiation could be attributed to crosslinks occurring in the boundaries between the crystalline and amorphous lamellae. If so, the increase in the transition width by $36 \AA$ at $400 \mathrm{Mrad}$ would cause a decrease in crystallinity to $20 \%$, assuming only half of the transition width contributes to a decrease of chain length in the crystalline phase. This value is much greater than the observèd $4 \%$ decrease in crystallinity. Therefore, this type of transition model is not applicable for crosslinked polyethylene and we may assume the existence of a sharp transition, or at least a constant transition width in the irradiated samples. Consequently, the decrease in $\left\langle\eta^{2}\right\rangle$ can only be attributed to a decrease in the denstiy difference between phases, suggesting a decrease in $\rho_{c}$ and/or an increase in $\rho_{a}$. This led to our reported values of $\rho_{c}$ and $\rho_{a}$ in the Results Section. Furthermore, changes in the crystalline and amorphous densities can remove the discrepancy between the crystallinities determined from WAXS and density data, which strongly supports the contention that the decrease in $\left\langle\eta^{2}\right\rangle$ is basically $\Delta \rho$ in origin.

The crystalline density $\rho_{c}$ remains essentially constant for linear and branched PE, at a value slightly lower than the ideal value $(0.993$ and 0.983 compared to 0.998), suggesting the presence of defects in the original crystal structure, expecially for the BPE. In contrast, the amorphous density $\rho_{a}$ increases with increasing radiation. The observed increase in $\rho_{a}$ with radiation suggests that crosslinks occur primarily outside the crystals in the amorphous phase. Crosslinks within the crystals would cause defects, leading to a continuous decrease in $\rho_{c}$ that is not observed in either BPE or LPE. The present findings give additional confirmation to our previous results on Alathon 10 obtained by photographic methods, as well as the conclusions reached there.

\section{Conclusions}

The use of SAXS to determine the structural changes in PE caused by radiation-induced crosslinking was confirmed. The crystalline density remains essentially unchanged, whereas the amorphous density increases with increasing radiation dose for both branched and linear PE. These results indicate that a simple two-phase model, assuming constant $\rho_{c}$ and 
$\rho_{a r}$ is not valid for the crosslinked PE. The results also explain the discrepancy between crystallinities determined from WAXS and density measurements. The constant values of crystalline density suggests no crosslinks form within crystals; crosslinking takes place primarily in a non-crystalline phase.

\section{Acknowledgement}

The authors wish to thank the Macromolecular Research Center and the National Science Foundation for financial support.

\section{References}

1. Yeh GSY, Chen CJ, Boose DC (1985) Colloid \& Polymer Sci 263:109

2. Fischer EW, Goddar H, Schmidt GF (1967) J Polym Sci B5:619

3. Vonk CG, Kortleve G (1967) Kolloid Z Polym 220:19

4. Kortleve G, Vonk CG (1968) Kolloid Z Polym 225:124

5. Fischer EW, Goddar H, Schmidt GF (1969) J Polym Sci A2, I, 37

6. Kavesh S, Schultz JM (1971) J Polym Sci A2, 9:85

7. Fischer EW, Fakirov S (1976) J Mater Sci 11:1041

8. Debye P, Bueche A (1949) J Appl Phys 20:518
9. Debye P, Anderson HR, Bueche A (1957) J Appl Phys 28:679

10. Porod G (1951) Kolloid Z 124:83

11. Cullity BD (1967) Elements of X-Ray Diffraction, AddisonWesley, Mass

12. Schmidt PW (1965) Acta Cryst 19:938

13. Flory PJ Principles of Polymer Chemistry, Cornell Univ Press, New York

14. Chen CJ, unpublished

15. Schmidt PW (1965) Acta Cryst 19:938

16. Strobl GR, Muller N (1973) Polym Sci Phys 11:1219

17. Tsvankin DY (1964) Polym Sci USSR 6:2304-2310

Received May 8, 1990 accepted July 4, 1990
Authors'address:

G. S. Y. Yeh

Departments of Chemical Engineering and Materials Engineering

Macromolecular Research Center

Univrsity of Michigan

Ann Arbor, Michigan 48109, USA 